Maslyuchenko Volodymyr, Department of Mathematical Analysis, Chernivtsi National University, 58012 Chernivtsi, Ukraine.

email: math.analysis.chnu@gmail.com

Nesterenko Vasyl', Department of Mathematical Analysis, Chernivtsi

National University, 58012 Chernivtsi, Ukraine.

email: math.analysis.chnu@gmail.com

\title{
ANALOGUES OF TRANSITIVENESS AND DECOMPOSITION OF CONTINUITY
}

\begin{abstract}
We consider two conditions that weaken the closed graph condition and we study their properties. We show that if $X$ is a locally connected Baire space, $Y$ is a separable metrizable space and $f: X \rightarrow Y$ is a $w^{*}$ quasi-continuous, almost continuous and weakly Darboux function, then $f$ is continuous.
\end{abstract}

\section{Introduction}

Issues related to the decomposition of continuity have been studied in the works of many mathematicians. Smith showed in [19] that the function $f$ : $[0,1] \rightarrow \mathbb{R}$ is continuous if and only if $f$ is almost continuous in the sense of Stallings, almost continuous in the sense of Husain and not of the Cesaro type. Later, J. Smital and E. Stanova generalized in [18] this result to the case of functions $f: X \rightarrow \mathbb{R}$, where $X$ is a locally connected Baire topological $T_{3}$-space. Finally, R. Gibson replaced in [5] the almost continuity in the sense of Stallings with the Darboux property and obtained the following result.

Theorem A. Let $X$ be a locally connected Baire topological $T_{3}$-space. A function $f: X \rightarrow \mathbb{R}$ is continuous if and only if

\footnotetext{
Mathematical Reviews subject classification: Primary: 54C30, 26A15

Key words: decomposition of continuity, closed graph, transitional mapping, locally $w^{*}$ continuous, locally relative continuous, almost continuity, Darboux properties

Received by the editors September 15, 2014

Communicated by: Krzysztof Ciesielski
} 
(1) $f$ is a Darboux function,

(2) $f$ is almost continuous in the sense of Husain, and

(3) $f$ is not of Cesaro type.

In [13], R. Mimna generalized a well-known result on the continuity of the function $f: \mathbb{R} \rightarrow \mathbb{R}$ with connected and closed graph. He established, using the concepts of $O$-connectedness and local $w^{*}$ continuity, the following result: if $X$ is a locally connected space and $Y$ a topological space, then the map $f: X \rightarrow Y$ is continuous if and only if $f$ is $O$-connected and locally $w^{*}$ continuous. Introducing the concept of transitiveness, V. Maslyuchenko and V. Kretsu proved in [8] that a function $f: \mathbb{R} \rightarrow \mathbb{R}$ is continuous if and only if $f$ is almost continuous in the sense of Stallings and transitional. The result of Mimna from [13] has been improved by V. Maslyuchenko and V. Nesterenko in [11] as the following theorem.

Theorem B. Let $X$ be a locally connected space and $Y$ a topological space. A function $f: X \rightarrow Y$ is continuous if and only if

1) $f$ is a weakly Darboux function,

2) $f$ is transitional.

Introducing the concept of $w^{*}$ quasi-continuity, M. Matejdes obtained in [12] another outcome to this thread.

Theorem C. Let $X$ be a $\pi$-connected space, $Y$ a topological space and $f$ : $X \rightarrow Y$ an $O$-connected function. If $f$ is almost continuous at $x$ and $w^{*}$ quasicontinuous at $x$, then $f$ is quasi-continuous at $x$.

If in Theorem $\mathrm{C}$ the space $Y$ is regular, then using the well-known decomposition theorem of [16], we obtain that the function $f$ is continuous.

In all of these results, when $Y$ is a locally compact Hausdorff space, the properties studied (e.g., transitiveness, local $w^{*}$ continuity and $w^{*}$ quasicontinuity of a function) are all strictly weaker than the closed graph property. These properties of functions are called analogs of transitiveness. Relations between them are studied in sections 3 and 4 .

Later in this article, we find that if $Y$ is a second countable space and $f: X \rightarrow Y$ is $w^{*}$ quasi-continuous, then the set of points of transitiveness of $f$ is residual in $X$; see Theorem 16. We also get a local version of Theorem B; see Theorem 18. With these tools, we obtain Theorem 20, a result on the set of points of discontinuity of continuous mappings that have the weak Darboux property, and Theorems 22 and 24, which develop Theorem C. 


\section{Definitions}

A function $f: X \rightarrow \mathbb{R}$ is said to be upper $\{$ lower $\}$ transitional at a point $x \in X$ [11] if, for any $\varepsilon>0$, there is a neighborhood $U$ of $x$ and a point $y \in(f(x), f(x)+\varepsilon)\{y \in(f(x)-\varepsilon, f(x))\}$ such that $U \cap f^{-1}(y)=\varnothing$. If a function is upper transitional and lower transitional at a point $x$, then the function is called transitional at $x$. A function is said to be transitional, upper transitional or lower transitional if it is so at each point.

For a subset $A$ of a topological space, let int $A, \bar{A}$ and fr $W=\bar{W} \backslash \operatorname{int} W$ denote the interior, the closure and the boundary of $A$, respectively. Let $X$ and $Y$ be topological spaces. A function $f: X \rightarrow Y$ is called

- transitional at $x \in X$ [11] if, for each neighborhood $V$ of $f(x)$ in $Y$, there is a neighborhood $U$ of $x$ in $X$ and an open neighborhood $W$ of $f(x)$ in $Y$ such that $W \subseteq V$ and $U \cap f^{-1}($ fr $W)=\varnothing$;

- weakly transitional at $x \in X[11]$ if, for each neighborhood $V$ of $f(x)$ in $Y$, there is a neighborhood $U$ of $x$ in $X$ and a point $b \in V$ such that $U \cap f^{-1}(b)=\varnothing$;

- quasi-transitional at $x \in X$ [11] if, for each neighborhood $V$ of $f(x)$ in $Y$ and each neighborhood $U$ of $x$ in $X$, there is an open neighborhood $W$ of $f(x)$ in $Y$ and a nonempty open subset $G$ of $X$ such that $W \subseteq V$, $G \subseteq U$ and $G \cap f^{-1}($ fr $W)=\varnothing ;$

- weakly quasi-transitional at $x \in X$ [11] if, for each neighborhood $V$ of $f(x)$ in $Y$ and each neighborhood $U$ of $x$ in $X$, there is a nonempty open set $G$ in $X$ and a point $b \in V$ such that $G \subseteq U$ and $G \cap f^{-1}(b)=\varnothing$.

A function is said to be transitional, weakly transitional, quasi-transitional, weakly quasi-transitional if it is so at each point. The class of transitional maps is very extensive. In particular, every continuous mapping is transitional, every monotone function $f: \mathbb{R} \rightarrow \mathbb{R}$ or a function with closed graph is transitional. It is clear that a function $f: X \rightarrow \mathbb{R}$ is weakly transitional at $x$ if and only if $f$ is upper or lower transitional at $x$. Obviously, if a mapping is transitional at some point, then it is quasi-transitional at that point.

Similar to as was introduced the notion of the upper or lower transitiveness of function $f: X \rightarrow \mathbb{R}$ can introduce the concept of upper or lower quasitransitiveness.

A set $A$ is quasi-open if $A \subseteq \overline{\operatorname{int} A}$. A function $f: X \rightarrow Y$ is said to be

- $w^{*}$ continuous [9] if $f^{-1}($ fr $V)$ is closed in $X$ for each open set $V$ in $Y$; 
- locally $w^{*}$ continuous [14] if there is a base $\mathcal{B}$ for $Y$ such that $f^{-1}(\operatorname{fr} B)$ is closed for any $B \in \mathcal{B}$;

- locally relative continuous [15] if there is a base $\mathcal{B}$ for $Y$ such that $f^{-1}(B)$ is an open set of a subspace $f^{-1}(\bar{B})$ for any $B \in \mathcal{B}$;

- $w^{*}$ quasi-continuous at $x \in X[12]$ if, for each neighborhood $V$ of $f(x)$ in $Y$, there is an open neighborhood $W$ of $f(x)$ in $Y$ and a quasi-open subset $A$ of $X$ such that $W \subseteq V, x \in A$ and $A \cap f^{-1}$ (fr $\left.W\right)=\varnothing$; and $w^{*}$ quasi-continuous if $f$ is so at every point.

It is said that $f: X \rightarrow Y$ is of the Cesaro type if there exist nonempty open subsets $U$ of $X$ and $V$ of $Y$ such that $f^{-1}(y)$ is dense in $U$ for each $y \in V$. In [11], it is shown that a function $f: X \rightarrow Y$ between topological spaces $X$ and $Y$ is weakly quasi-transitional if and only if $f$ is not of Cesaro type.

Denote by $\operatorname{Gr}(f)=\{(x, f(x)): x \in X\}$ the graph of $f$. A function $f: X \rightarrow Y$ is called

- almost continuous in the sense of Stallings [20] if, for any open subset $O$ of $X \times Y$ containing the graph of $f, O$ contains the graph of a continuous function $g: X \rightarrow Y$;

- a Darboux function [2] if $f(A)$ is a connected subset of $Y$ whenever $A$ is a connected subset of $X$;

- a weakly Darboux function [11] (also O-connected [13]) if $f(G)$ is a connected subset of $Y$ whenever $G$ is an open connected subset of $X$;

- almost continuous in the sense of Husain at $x \in X[3,6,17]$ if, for each neighborhood $V$ of $f(x)$ in $Y$, there is a subset $A$ of $X$ such that $x \in \operatorname{int} \bar{A}$ and $f(A) \subseteq V$; and almost continuous in the sense of Husain if $f$ is so at any point;

- quasi-continuous at $x \in X[7,12]$ if, for each neighborhood $V$ of $f(x)$ in $Y$ and each neighborhood $U$ of $x$ in $X$, there is a nonempty open subset $G$ of $X$ such that $G \subseteq U$ and $f(G) \subseteq V$; and quasi-continuous if $f$ is so at any point.

\section{Relations between different analogues of transitiveness which are stronger than transitiveness}

First we note that local $w^{*}$ continuity is a weaker condition than $w^{*}$ continuity. This is shown by the following example. 
Example 1. The function $f: \mathbb{R} \rightarrow \mathbb{R}$ defined as

$$
f(x)= \begin{cases}1, & x \in \mathbb{Q} \\ 0, & x \notin \mathbb{Q}\end{cases}
$$

is locally $w^{*}$ continuous, but is not $w^{*}$ continuous.

In [11, Theorem 17], it was established that every locally $w^{*}$ continuous function $f: X \rightarrow Y$ between topological spaces $X$ and $Y$ is transitional. [11, example 3] shows that the inverse statement is not true.

In [15], it was established that for arbitrary topological spaces $X$ and $Y$, a locally $w^{*}$ continuous function $f: X \rightarrow Y$ is locally relative continuous. There is also an example of a function $f: \mathbb{R} \rightarrow \mathbb{R}$ which shows that the converse statement is not true.

Proposition 2. Let $X$ and $Y$ be topological spaces and $f: X \rightarrow Y$ a locally relatively continuous function. Then $f$ is transitional.

Proof. Assume the contrary, that $f$ is not transitional at $x_{0}$. Then there is a neighborhood $V_{0}$ of $f\left(x_{0}\right)$ in $Y$ such that for each open neighborhood $U$ of $x_{0}$ in $X$ and for each open neighborhood $W$ of $f\left(x_{0}\right)$ in $Y$ with $W \subseteq V_{0}$, we have that $U \cap f^{-1}($ fr $W) \neq \varnothing$.

Since $f$ is locally relatively continuous, there is a base $\mathcal{B}$ of open subsets of $Y$ such that $f^{-1}(V)$ is an open subset of $f^{-1}(\bar{V})$ for each $V \in \mathcal{B}$. For the point $f\left(x_{0}\right)$ and a neighborhood $V_{0}$ of $f\left(x_{0}\right)$, there is $V \in \mathcal{B}$ such that $f\left(x_{0}\right) \in V \subseteq V_{0}$. We take an arbitrary neighborhood $U$ of $x_{0}$ in $X$. Since $f$ is not transitional at $x_{0}, U \cap f^{-1}(\operatorname{fr} V) \neq \varnothing$. Then there is a point $x \in U$ such that

$$
x \in f^{-1}(\operatorname{fr} V)=f^{-1}(\bar{V} \backslash V)=f^{-1}(\bar{V}) \backslash f^{-1}(V) .
$$

Then $U \cap f^{-1}(\bar{V}) \nsubseteq f^{-1}(V)$. This means that $x_{0}$ is not an interior point of $f^{-1}(V)$ in the subspace $f^{-1}(\bar{V})$. Thus, $f^{-1}(V)$ is not an open subset of $f^{-1}(\bar{V})$. This contradicts the fact that $f$ is locally relatively continuous. So our assumption is not true.

As already noted, a transitional mapping is not required to be locally $w^{*}$ continuous [11, Example 3]. The same example shows that a transitional mapping is not required to be locally relatively continuous.

Proposition 3. There is a transitional function $f: \mathbb{R} \rightarrow \mathbb{R}$ which is not locally relatively continuous. 
Proof. Let $A=(-\infty,-1]=\bigsqcup_{r \in \mathbb{Q}} A_{r}$, where $A_{r}$ is dense in $A$ for each $r \in \mathbb{Q}$, and $B=[1,+\infty)=\bigsqcup_{\xi \in \mathbb{R} \backslash \mathbb{Q}} B_{\xi}$, where $B_{\xi}$ is dense in $B$ for each $\xi \in \mathbb{R} \backslash \mathbb{Q}$. Let $f: \mathbb{R} \rightarrow \mathbb{R}$ be defined as

$$
f(x)=\left\{\begin{array}{ll}
r, & x \in A_{r} \\
\xi, & x \in B_{\xi} \\
0, & x \in(-1,1)
\end{array} .\right.
$$

The function $f$ is transitional at an arbitrary point $x \in \mathbb{R}$. It is clear for points in the interval $(-1,1)$, because $f$ is constant on this interval. If $x \geq 1$ and $\varepsilon>0$, then there are rational numbers $y_{1} \in(f(x), f(x)+\varepsilon)$ and $y_{2} \in(f(x)-\varepsilon, f(x))$ such that

$$
\left(U \times\left\{y_{i}\right\}\right) \cap \operatorname{Gr}(f)=\varnothing, \quad i=1,2
$$

for the neighborhood $U=(0,+\infty)$ of $x$. If $x \leq-1$, then for irrational numbers $y_{1} \in(f(x), f(x)+\varepsilon)$ and $y_{2} \in(f(x)-\varepsilon, f(x))$ and the neighborhood $U=$ $(-\infty, 0)$, we have that $\left(U \times\left\{y_{i}\right\}\right) \cap \operatorname{Gr}(f)=\varnothing, i=1,2$.

Let $\mathcal{B}$ be an arbitrary base of $\mathbb{R}$. Then there is a set $V \in \mathcal{B}$ such that $\varnothing \neq V \subseteq(1,+\infty)$. We show that $f^{-1}(V)$ is not an open subset of $f^{-1}(\bar{V})$.

First, we note that $\bar{V} \backslash V \neq \varnothing$. Indeed $\varnothing \neq V \subseteq \bar{V} \subseteq[1,+\infty) \subset \mathbb{R}$. If $\bar{V}=V$, then $V$ is a open-closed subset of $\mathbb{R}, V \neq \varnothing$ and $V \neq \mathbb{R}$. This contradicts the connectedness of $\mathbb{R}$.

So there is $y \in \bar{V} \backslash V$. It is clear that $y \neq 0$ because $\bar{V} \subseteq[1,+\infty)$.

Suppose that $y \in \mathbb{Q}$. Then $f^{-1}(y)=A_{y}$. By construction, we have that $\overline{A_{y}}=(-\infty,-1]$. We take a rational number $y_{0}$ in $V$ and consider an arbitrary point $x_{0}$ in $f^{-1}\left(y_{0}\right)=A_{y_{0}}$. Then $x_{0} \in f^{-1}(V)$. We show then $x_{0}$ is not a interior point of the subset $f^{-1}(V)$ of $f^{-1}(\bar{V})$. Let $U$ be an arbitrary neighborhood of $x_{0}$ in $\mathbb{R}$. Since $x_{0} \in A_{y_{0}} \subseteq(-\infty,-1]$ and $\overline{A_{y}} \supseteq(-\infty,-1]$, $x_{0} \in \overline{A_{y}}$. So $U \cap A_{y} \neq \varnothing$, namely there is $u \in U \cap A_{y}$. Then $u \in U \cap f^{-1}(\bar{V})$ and $u \notin f^{-1}(V)$ because $f(u)=y \notin V$. Hence, $U \cap f^{-1}(\bar{V}) \nsubseteq f^{-1}(V)$ for each neighborhood $U$ of $x_{0} \in f^{-1}(V)$. This shows that $f^{-1}(V)$ is not an open subset of $f^{-1}(\bar{V})$.

If $y \in \mathbb{R} \backslash \mathbb{Q}$, then $f^{-1}(y)=B_{y}$. By construction, we have that $\overline{B_{y}}=$ $[1,+\infty)$. Take a irrational number $y_{0} \in V$ and consider a point $x_{0} \in f^{-1}\left(y_{0}\right)=$ $B_{y_{0}}$. It is then easy to verify that $U \cap f^{-1}(\bar{V}) \nsubseteq f^{-1}(V)$ for any neighborhood $U$ of $x_{0}$ in $\mathbb{R}$. Hence, $f^{-1}(V)$ is not an open subset of $f^{-1}(\bar{V})$. 


\section{Relations between different analogues of transitiveness which are weaker than transitiveness}

Proposition 4. Let $X$ and $Y$ be topological spaces and $f: X \rightarrow Y$ a function which is transitional at a point $x_{0} \in X$. Then $f$ is $w^{*}$ quasi-continuous at $x_{0}$.

Proof. Let $V$ be any neighborhood of $y_{0}=f\left(x_{0}\right)$ in $Y$. Since $f$ is transitional at $x_{0}$, there is a neighborhood $U$ of $x_{0}$ in $X$ and an open neighborhood $W$ of $y_{0}$ in $Y$ such that $U \cap f^{-1}(\operatorname{fr} W)=\varnothing$. The open set $G=\operatorname{int} U$ is a neighborhood of $x_{0}$ in $X$ and $G \cap f^{-1}$ (fr $\left.W\right)=\varnothing$ with $G \subseteq U$. The open set $G$ is quasi-open in $X$; hence, $f$ is $w^{*}$ quasi-continuous at $x_{0}$.

In [11], it was shown that every continuous mapping is transitional. A similar result holds for the $w^{*}$ quasi-continuity.

Proposition 5. Let $X$ and $Y$ be topological spaces and $f: X \rightarrow Y$ a function which is quasi-continuous at a point $x \in X$. Then $f$ is $w^{*}$ quasi-continuous at $x$.

Proof. Take any neighborhood $V$ of $f(x)$ in $Y$. Put $W=\operatorname{int} V$. It is clear that $W \subseteq V$ and $W$ is an open neighborhood of $f(x)$ in $Y$. Since $f$ is quasicontinuous at $x$, for each neighborhood $U$ of $x$ there is a nonempty open subset $G_{U}$ of $X$ such that $G_{U} \subseteq U$ and $f\left(G_{U}\right) \subseteq$ int $W$. Consider the set $H=\bigcup\left\{G_{U}: U\right.$ is a neighborhood of $\left.x\right\}$. It is clear that $x \in \bar{H}$. It is easy to verify that $A=H \cup\{x\}$ is quasi-open in $X$. In addition, $x \in A, f(A) \subseteq W$ and $W \cap$ fr $W=\varnothing$, because $W$ is an open subset of $Y$. Hence, $A \cap f^{-1}(\mathrm{fr} W)=\varnothing$. This shows that $f$ is $w^{*}$ quasi-continuous at $x$.

Obviously, the converse is not true. In fact, the Dirichlet function is transitional and thus $w^{*}$ quasi-continuous, but is not quasi-continuous at any point in $\mathbb{R}$.

Also, the converse to Proposition 4 is not true.

Example 6. The function $f: \mathbb{R} \rightarrow \mathbb{R}$ defined as

$$
f(x)=\left\{\begin{array}{ll}
\sin \frac{1}{x}, & x \neq 0 \\
0, & x=0
\end{array} .\right.
$$

is $w^{*}$ quasi-continuous because $f$ is quasi-continuous; see Proposition 5. But $f$ is not transitional.

Proposition 7. Let $X$ and $Y$ be topological spaces and $f: X \rightarrow Y$ a function which is $w^{*}$ quasi-continuous at a point $x \in X$. Then $f$ is quasi-transitional at $x$. 
Proof. Take arbitrary neighborhoods $V$ of $f(x)$ and $U$ of $x$ in $Y$ and $X$, respectively. Since $f$ is $w^{*}$ quasi-continuous at $x$, there is an open neighborhood $W$ of $f(x)$ in $Y$ and a quasi-open subset $A$ of $X$ such that $W \subseteq V, x \in A$ and $A \cap f^{-1}$ (fr $\left.W\right)=\varnothing$. The set $A$ is quasi-open; therefore, $x \in A \subseteq \overline{\operatorname{int} A}$. Since $U$ is a neighborhood of $x, G=U \cap \operatorname{int} A \neq \varnothing$. The set $G$ is open and $G \subseteq U$. Since $A \cap f^{-1}($ fr $W)=\varnothing, G \cap f^{-1}($ fr $W)=\varnothing$. This means that $f$ is quasi-transitional at $x$.

In Proposition 12, we will show that there exists a function $f: \mathbb{R} \rightarrow \mathbb{R}$ which is quasi-transitional but not $w^{*}$ quasi-continuous.

In [11, Theorem 7], conditions are given for spaces $X$ and $Y$ in which $f: X \rightarrow Y$ is transitional at $x$ implies $f$ is weakly transitional at $x$. The same conditions on spaces ensure that a mapping is weakly quasi-transitional at a point, provided that this mapping is quasi-transitional at that point.

Proposition 8. Let $X$ be a topological space, $Y$ a regular space, $x \in X$ and $f: X \rightarrow Y$ a function which is quasi-transitional at $x$ such that there is a connected neighborhood $V_{0}$ of $y=f(x)$ in $Y$ with $V_{0} \neq\{y\}$. Then $f$ is weakly quasi-transitional at $x$.

Proof. Suppose that $V$ is a neighborhood of $y$ in $Y$ and $U$ is a neighborhood of $x$ in $X$. By assumption, there is a point $y_{0} \in V_{0}$ such that $y_{0} \neq y$. It follows from $T_{1}$-axiom that there is a neighborhood $V_{1}$ of $y$ in $Y$ such that $y_{0} \notin V_{1}$. Since $Y$ is regular space, there is a closed neighborhood $V_{2}$ of $y$ in $Y$ such that $V_{2} \subseteq V \cap V_{0} \cap V_{1}$. It follows from the quasi-transitiveness of $f$ that there is a nonempty open subset $G$ of $X$ and an open neighborhood $W$ of $y$ in $Y$ such that $W \subseteq V_{2}, G \subseteq U$ and $G \cap f^{-1}$ (fr $\left.W\right)=\varnothing$. We show that fr $W \neq \varnothing$. If fr $W=\varnothing$, then $W$ is an open-closed subset of $Y$ and $W \subseteq V_{0}$. Moreover, since $y_{0} \in V_{0} \backslash W$, we obtain $W \subset V_{0}$. In addition, $W \neq \varnothing$ because $y \in W$. Then the connected set $V_{0}$ is the disjoint union of two nonempy sets $W$ and $V_{0} \backslash W$, each open in $V_{0}$, which is impossible.

So fr $W \neq \varnothing$, and hence, there is a point $b \in \operatorname{fr} W$. Since $W \subseteq V_{2}$ and $V_{2}$ is closed set, fr $W \subseteq V_{2} \subseteq V$. In this case, $b \in V$ and $b \notin f(G)$. Hence, $G \cap f^{-1}(b)=\varnothing$. This means that $f$ is weakly quasi-transitional at $x$.

In [11, Theorem 1], it was established that if $\mathbb{R} \backslash f(X)$ is an everywhere dense set for the function $f: X \rightarrow \mathbb{R}$, then $f$ is transitional.

Proposition 9. Let $X$ and $Y$ be topological spaces and $f: X \rightarrow Y$ a function such that $\overline{Y \backslash f(X)}=Y$. Then $f$ is weakly transitional.

Proof. Let $x \in X$ and $V$ a neighborhood of $f(x)$ in $Y$. Since $Y \backslash f(X)$ is an everywhere dense subset of $Y$, there is a point $b \in V \cap(Y \backslash f(X))$. Therefore, $X \cap f^{-1}(b)=\varnothing$. Hence, $f$ is weakly transitional at $x$. 
Proposition 10. Let $X$ be a topological space. Then each weakly quasitransitional function $f: X \rightarrow \mathbb{R}$ is quasi-transitional.

Proof. Let $x_{0} \in X, y_{0}=f\left(x_{0}\right), U$ an open neighborhood of $x_{0}$ in $X$ and $V=\left(y_{0}-\varepsilon, y_{0}+\varepsilon\right)$ a neighborhood of $y_{0}$. Suppose that there is a point $x_{1} \in U$ such that $f\left(x_{1}\right) \in V_{1}=\left(y_{0}, y_{0}+\varepsilon\right)$. Since $f$ is weakly transitional at $x_{1}$, there is a nonempty open subset $G_{1}$ of $X$ and a point $b_{1} \in V_{1}$ such that $G_{1} \subseteq U$ and $b_{1} \notin f\left(G_{1}\right)$. Otherwise, for any point $y \in\left(y_{0}, y_{0}+\varepsilon\right)$, we obtain $y \notin f(U)$. Anyway, there is a nonempty open set $G_{1} \subseteq U$ and a point $b_{1} \in V_{1}$ such that $b_{1} \notin f\left(G_{1}\right)$. Similarly, we establish that there is a nonempty open set $G_{2} \subseteq G_{1}$ and a point $b_{2} \in V_{2}=\left(y_{0}-\varepsilon, y_{0}\right)$ such that $b_{2} \notin f\left(G_{2}\right)$.

The interval $W=\left(b_{2}, b_{1}\right)$ is a neighborhood of $f\left(x_{0}\right)$ in $\mathbb{R}, W \subseteq V$ and $G_{2} \cap f^{-1}($ fr $W)=G_{2} \cap f^{-1}\left(\left\{b_{2}, b_{1}\right\}\right)=\varnothing$. Hence, $f$ is quasi-transitional at $x_{0}$. This means that $f$ is quasi-transitional.

Note that the function in Proposition 10 is real-valued; the following proposition shows that this is an important hypothesis.

Proposition 11. There is a weakly transitional function $f: \mathbb{R} \rightarrow \mathbb{R}^{2}$ (hence, weakly quasi-transitional) which is not quasi-transitional at any point.

Proof. Let $\left(A_{\alpha}\right)_{\alpha \in \mathbb{R}}$ be a system of disjoint everywhere dense subsets of $\mathbb{R}$ such that $\bigsqcup_{\alpha \in \mathbb{R}} A_{\alpha}=\mathbb{R}$. The function $f: \mathbb{R} \rightarrow \mathbb{R}^{2}$ defined as $f(x)=(\alpha, 0)$, $x \in A_{\alpha}$ is weakly transitional, but is not quasi-transitional at any point.

In fact, since $f(\mathbb{R})=\mathbb{R} \times\{0\}$, by Proposition 9 it follows that $f$ is weakly transitional. Take any point $x \in \mathbb{R}$ and a bounded neighborhood $V$ of $f(x)$ in $\mathbb{R}^{2}$. Since $\mathbb{R} \times\{0\}$ is a connected subset of $\mathbb{R}^{2}$, fr $W \cap(\mathbb{R} \times\{0\}) \neq \varnothing$ for an arbitrary open neighborhood $W$ of $f(x)$ in $\mathbb{R}^{2}$ such that $W \subseteq V$. Let $(\alpha, 0) \in$ fr $W \cap(\mathbb{R} \times\{0\})$. Then

$$
f^{-1}(\text { fr } W) \supseteq f^{-1}((\alpha, 0))=A_{\alpha},
$$

and therefore, $f^{-1}(\operatorname{fr} W)$ is an everywhere dense subset of $\mathbb{R}$. Hence, $f$ is not quasi-transitional at $x$.

Proposition 12. There is a function $f: \mathbb{R} \rightarrow \mathbb{R}$ which is quasi-transitional, but not $w^{*}$ quasi-continuous.

Proof. Again, let $\left(A_{\alpha}\right)_{\alpha \in \mathbb{R}}$ be a system of disjoint everywhere dense subsets of $\mathbb{R}$ such that $\bigsqcup_{\alpha \in \mathbb{R}} A_{\alpha}=\mathbb{R}$. Let $f_{0}$ be the function of $\mathbb{R}$ to $\mathbb{R}$ such that $f_{0}(x)=\alpha$ for $x \in A_{\alpha}$. Note that $f_{0}^{-1}(\alpha)=A_{\alpha}$ is an everywhere dense subset for any point $\alpha \in \mathbb{R}$; hence, $f_{0}$ is not weakly quasi-transitional at any point. 
Let $\mathbb{Q}=\left\{r_{n}: n \in \mathbb{N}\right\}$ be the set of nonzero rational numbers. Let the function $f: \mathbb{R} \rightarrow \mathbb{R}$ be given by

$$
f(x)=\left\{\begin{array}{ll}
0, & x \in A_{r_{n}} \backslash\left(-\frac{1}{n}, \frac{1}{n}\right) \\
f_{0}(x), & \text { otherwise }
\end{array} .\right.
$$

Let us prove that $f$ is quasi-transitional, but not $w^{*}$ quasi-continuous. Take any point $x \in \mathbb{R}$, a neighborhood $U$ of $x$ and a neighborhood $V$ of $f(x)$. There is a positive integer $n$ such that $r_{n} \in V$ and $G=\operatorname{int}\left(U \backslash\left(-\frac{1}{n}, \frac{1}{n}\right)\right) \neq \varnothing$. Then $\left.G \cap f^{-1}\left(r_{n}\right)\right)=\varnothing$. So, $f$ is weakly quasi-transitional at $x$. Therefore, $f$ is weakly quasi-transitional, and by proposition 10, it follows that $f$ is quasitransitional.

We show that $f$ is not $w^{*}$ quasi-continuous at 0 . Consider the neighborhood $V=\left(y_{0}-1, y_{0}+1\right)$ of $y_{0}=f(0)$ and an arbitrary open neighborhood $W$ of $y_{0}$ such that $W \subseteq V$. Take any quasi-open set $A$ such that $0 \in A$. We show that $A \cap f^{-1}($ fr $W) \neq \varnothing$. Note that if fr $E=\varnothing$ for $E \subseteq \mathbb{R}$, then $\bar{E}=\operatorname{int} E$. So $E$ is open-closed in $\mathbb{R}$. Therefore, $E=\varnothing$ or $E=\mathbb{R}$, because $\mathbb{R}$ is a connected space. But $\varnothing \neq W$ and $W \neq \mathbb{R}$. Hence, fr $W \neq \varnothing$, and so there is a point $b \in$ fr $W$.

Suppose that $b=r_{n}$ for some $n$. Since $A \subseteq \overline{\operatorname{int} A}, 0 \in A$ and $\left(-\frac{1}{n}, \frac{1}{n}\right)$ is a neighborhood 0 in $\mathbb{R}, U_{n}=\left(-\frac{1}{n}, \frac{1}{n}\right) \cap \operatorname{int} A \neq \varnothing$. The set $A_{r_{n}}$ is dense in $\mathbb{R}$; therefore, $U_{n} \cap A_{r_{n}} \neq \varnothing$. But $\left(-\frac{1}{n}, \frac{1}{n}\right) \cap A_{r_{n}}=f^{-1}\left(r_{n}\right)$; therefore, $f^{-1}\left(r_{n}\right) \cap \operatorname{int} A \neq \varnothing$. So $f^{-1}\left(r_{n}\right) \cap A \neq \varnothing$. Then there is a point $a \in A$ such that $f(a)=r_{n}=b \in$ fr $W$. Hence, $A \cap f^{-1}($ fr $W) \neq \varnothing$.

Let $b \in(\mathbb{R} \backslash \mathbb{Q}) \cup\{0\}$ be fixed. Since $A_{b}$ is dense in $\mathbb{R}$ and int $A \neq \varnothing$, $\operatorname{int} A \cap A_{b} \neq \varnothing$. So, there is a point $a \in A$ such that $a \in A_{b}$. But $b \neq r_{n}$ for each $n$. Hence, $A_{b} \cap A_{r_{n}}=\varnothing$ for each $n$. Therefore, $f(a)=f_{0}(a)=b$. In this case, we have that $A \cap f^{-1}$ (fr $\left.W\right) \neq \varnothing$.

Hence, $f$ is not $w^{*}$ quasi-continuous at 0 .

So, for arbitrary topological spaces $X$ and $Y$, we have the following implications:

$$
\begin{gathered}
w^{*} \text { continuity } \Rightarrow \text { local } w^{*} \text { continuity } \Rightarrow \text { transitiveness } \Rightarrow \\
\Rightarrow w^{*} \text { quasi-continuity } \Rightarrow \text { quasi-transitiveness. }
\end{gathered}
$$

And none of these implications can not be reversed.

Proposition 13. If $X$ is a topological space and $Y$ has a basis consisting of clopen sets, then each mapping $f: X \rightarrow Y$ is transitional. 
Proof. Indeed, for any point $x \in X$ and any neighborhood $V$ of $y=f(x)$ in $Y$, there is a clopen neighborhood $W$ of $y$ in $Y$ such that $W \subseteq V$. Then for the neighborhood $U=X$ of $x$ in $X$, we have that $U \cap f^{-1}($ fr $W)=\varnothing$, because fr $W=\varnothing$. Hence, $f$ is transitional at $x$.

In particular, all functions $f: X \rightarrow Y$, where $\mathrm{Y}$ is the set of rational numbers $\mathbb{Q}$ or the Sorgenfrey plane, are transitional.

Proposition 14. Each function $f: \mathbb{Q} \rightarrow \mathbb{R}$ is transitional.

Proof. Take any point $x \in \mathbb{Q}$, a neighborhood $U=(x-\delta, x+\delta) \cap \mathbb{Q}$ of $x$ in $\mathbb{Q}$ and a neighborhood $V=(f(x)-\varepsilon, f(x)+\varepsilon)$ of $f(x)$ in $\mathbb{R}$. We put $V_{1}=(f(x), f(x)+\varepsilon)$. By the symbol $|E|$, we denote the power of a set $E$. Since $\aleph_{0}=|U|<\left|V_{1}\right|=\mathfrak{c}$, there is a point $y_{1} \in V_{1}$ such that $y_{1} \notin f(U)$. So, $f$ is upper transitional at $x$. Similarly, we prove that $f$ is lower transitional at $x$. Hence, $f$ is transitional at $x$.

\section{On the set of points of transitiveness}

In [11], the following results were established.

Theorem 15. If $X$ is a topological space, a space $Y$ has a countable pseudobase and $f: X \rightarrow Y$ is a weakly quasi-transitional mapping, then $\{x \in X: f$ is weakly transitional at $x\}$ is a residual subset of $X$.

Theorem 16. Let $X$ be a topological space and $f: X \rightarrow \mathbb{R}$ a weakly quasitransitional function. Then the set of points of transitiveness of $f$ is residual in $X$.

A similar result can be obtained for $w^{*}$ quasi-continuous mappings.

Theorem 17. Let $X$ be a topological space, $Y$ a second countable space and $f: X \rightarrow Y$ a $w^{*}$ quasi-continuous mapping. Then the set $A$ of points of transitiveness of $f$ is residual in $X$.

Proof. Let $\left\{V_{n}: n \in \mathbb{N}\right\}$ be a base of $Y$. Assume the contrary. Let $E=$ $X \backslash A$ be a non-meagre subset of $X$. Then for any point $x \in E$, there is a neighborhood $V(x)$ of $f(x)$ such that $f(U) \cap$ fr $W \neq \varnothing$ for each neighborhood $U$ of $x$ in $X$ and for each open neighborhood $W$ of $f(x)$ with $W \subseteq V(x)$.

For each $n \in \mathbb{N}$, consider the set

$$
E_{n}=\left\{x \in E: f(x) \in V_{n} \subseteq V(x)\right\} .
$$

It is clear that $\bigcup_{n=1}^{\infty} E_{n}=E$. Since $E$ is a non-meagre set, there is a positive integer $n_{0}$ such that $E_{n_{0}}$ is a non-meagre set. By $w^{*}$ quasi-continuity of $f$, it 
follows that for each point $x \in E_{n_{0}}$, there is an open neighborhood $W(x)$ of $f(x)$ in $Y$ and a quasi-open subset $A(x)$ of $X$ such that $W(x) \subseteq V_{n_{0}}, x \in A(x)$ and $A(x) \cap f^{-1}($ fr $W(x))=\varnothing$.

For each $m \in \mathbb{N}$, we consider sets

$$
E_{n_{0}, m}=\left\{x \in E_{n_{0}}: f(x) \in V_{m} \subseteq W(x)\right\} .
$$

Then $\bigcup_{m=1}^{\infty} E_{n_{0}, m}=E_{n_{0}}$. Since $E_{n_{0}}$ is non-meagre, there is a positive integer $m_{0}$ such that $E_{n_{0}, m_{0}}$ is dense in a nonempty open subset $U_{0}$; i.e., $U_{0} \subseteq \overline{E_{n_{0}, m_{0}}}$. Take a point $x_{0} \in U_{0} \cap E_{n_{0}, m_{0}}$. Since $A\left(x_{0}\right)$ is quasi-open, $U=U_{0} \cap \operatorname{int} A\left(x_{0}\right) \neq$ $\varnothing$. Take a point $a \in U \cap E_{n_{0}, m_{0}}$. Then $U$ is a neighborhood of $a$. Since $U \subseteq$ $A\left(x_{0}\right), f(a) \in V_{m_{0}} \subseteq W\left(x_{0}\right) \subseteq V_{n_{0}} \subseteq V(a)$ and $A\left(x_{0}\right) \cap f^{-1}\left(\right.$ fr $\left.W\left(x_{0}\right)\right)=\varnothing$, $U \cap f^{-1}\left(\right.$ fr $\left.W\left(x_{0}\right)\right)=\varnothing$. In addition, $W\left(x_{0}\right)$ is an open neighborhood of $f(a)$. Hence, for $a \in E$, we found the neighbohood $U$ of $a$ and the open neighborhood $W=W\left(x_{0}\right)$ of $a$ such that $W \subseteq V(a)$ and $U \cap f^{-1}(\operatorname{fr} W)=\varnothing$. We obtained a contradiction.

\section{Main results}

The following result is a local version of Theorem B.

Theorem 18. Let $X$ be a locally connected space, $Y$ a topological space and $f: X \rightarrow Y$ a weakly Darboux function. Then $f$ is continuous at $x_{0} \in X$ if and only if $f$ is transitional at $x_{0}$.

Proof. Since $f: X \rightarrow Y$ is continuous at $x_{0}, f$ is transitional at $x_{0}$ for arbitrary topological spaces $X$ and $Y$ [11, Theorem 5]. In fact, let $V$ be any neighborhood of $y_{0}=f\left(x_{0}\right)$ in $Y$. Put $W=\operatorname{int} V$ and $U=f^{-1}(W)$. Then fr $W=\bar{W} \backslash W, y_{0} \in W \subseteq V$ and $U$ is a neighborhood of $x_{0}$ in $X$. Thus,

$U \cap f^{-1}(\operatorname{fr} W)=f^{-1}(W) \cap f^{-1}(\bar{W} \backslash W)=f^{-1}(W \cap(\bar{W} \backslash W))=f^{-1}(\varnothing)=\varnothing$.

We establish the sufficiency. Since $f$ is transitional at $x_{0}$, there is a neighborhood $U$ of $x_{0}$ in $X$ and an open neighborhood $W$ of $f\left(x_{0}\right)$ in $Y$ such that $W \subseteq V$ and $f(U) \subseteq W \sqcup(Y \backslash \bar{W})$. There is an open connected neighborhood $U_{0}$ of $x_{0}$ such that $U_{0} \subseteq U$. This neighborhood will be a connected component of any open neighborhood $G$ of $x_{0}$ containing that point and contained in $U$. It is clear that $f\left(U_{0}\right) \subseteq W \sqcup(Y \backslash \bar{W})$. Since $f$ is a weakly Darboux function, we obtain that $f\left(U_{0}\right)$ is a connected set. Then from the condition $f\left(x_{0}\right) \in W$, it follows that $f\left(U_{0}\right) \subseteq W$. Hence, we obtain that $f\left(U_{0}\right) \subseteq V$. This means that $f$ is continuous at $x_{0}$. 
It is clear that Theorem 18 immediately implies Theorem B.

For a mapping $f: X \rightarrow Y$, we denote by $C(f)$ and $D(f)$ the set of continuity points of $f$ and the set of discontinuity points of $f$, respectively.

Theorem 19. Let $X$ be a locally connected space, $Y$ a second countable space and $f: X \rightarrow Y$ a $w^{*}$ quasi-continuous and weakly Darboux function. Then $D(f)$ is a meagre subset of $X$.

Proof. By Theorem 17, it follows that the set $E$ of points of transition of $f$ is a residual, and by Theorem 18, it follows that $C(f)=E$. Hence, $D(f)$ is a meagre subset.

Theorem 20. Let $X$ be a locally connected space and $f: X \rightarrow \mathbb{R}$ a weakly quasi-transitional and weakly Darboux function. Then $D(f)$ is meagre subset of $X$.

Proof. By Theorem 16, it follows that the set $E$ of points of transition $f$ is a residual, and by Theorem 18, it follows that $C(f)=E$. Hence, $D(f)$ is a meagre subset.

A function $f$ is called almost quasi-continuous at $x \in X$ [1], if for each neighborhood $V$ of $y=f(x)$ in $Y$ and for each neighborhood $U$ of $x$ in $X$, there is a subset $A$ of $X$ such that $A \subseteq U$, int $\bar{A} \neq \varnothing$ and $f(A) \subseteq V$; and almost quasi-continuous if $f$ is so at every point. Let $Y$ be a metric space. A function $f: X \rightarrow Y$, where $Y$ is equipped with metric $d$, is said to be cliquish at a point $x \in X[21]$ if, for any $\varepsilon>0$ and any neighborhood $U$ of $x \in X$, there exists a nonempty open subset $G$ of $X$ such that $G \subseteq U$ and $d(f(u), f(v))<\varepsilon$ for each $u, v \in G$; and cliquish if $f$ is so at every point. We need the following two results that are given for example in [4] and [1], respectively:

(1) for a metric space $Y$, a function $f: X \rightarrow Y$ is continuous if and only if $f$ is almost continuous in the sense of Husain and cliquish;

(2) for a metric space $Y$, a function $f: X \rightarrow Y$ is quasi-continuous if and only if $f$ is almost quasi-continuous and cliquish.

Recall that a function $f: X \rightarrow Y$ is called pointwise discontinuous if $\overline{C(f)}=X$. It is easy to verify that for a topological space $X$ and a metric space $Y$, each pointwise discontinuous function is cliquish.

Theorem 21. Let $X$ be a locally connected Baire space, $Y$ a separable metrizable space and $f: X \rightarrow Y$ a function satisfying

1) $f$ is $w^{*}$ quasi-continuous;

2) $f$ is a weakly Darboux function;

3) $f$ is almost continuous in the sense of Husain.

Then $f$ is continuous. 
Proof. Fix a metric on the space $Y$ which generates its topology. By Theorem 19, it follows that $C(f)$ is a residual set in $X$, and therefore, $C(f)$ is an everywhere dense subset. Then $f$ is pointwise discontinuous, and hence, $f$ is cliquish. By (1), it follows that $f$ is continuous.

Theorem 22. Let $X$ be a locally connected Baire space and $f: X \rightarrow \mathbb{R}$ a function satisfying

1) $f$ is weakly quasi-transitional;

2) $f$ is a weakly Darboux function;

3) $f$ is almost continuous in the sense of Husain.

Then $f$ is continuous.

Proof. By Theorem 20, it follows that $C(f)$ is a residual set in $X$. Since $X$ is a Baire space, $\overline{C(f)}=X$. Then $f$ is pointwise discontinuous, and hence, $f$ is cliquish. By (1), it follows that $f$ is continuous.

Theorem 23. Let $X$ be a locally connected Baire space, $Y$ a separable metrizable space and $f: X \rightarrow Y$ a function satisfying

1) $f$ is $w^{*}$ quasi-continuous;

2) $f$ is a weakly Darboux function;

3) $f$ is almost quasi-continuous.

Then $f$ is quasi-continuous.

Proof. Similar to in the proof of Theorem 21, one can establish that $f$ is cliquish. By (2), it follows that $f$ is quasi-continuous.

Theorem 24. Let $X$ be a locally connected Baire space and $f: X \rightarrow \mathbb{R} a$ function satisfying

1) $f$ is weakly quasi-transitional;

2) $f$ is a weakly Darboux function;

3) $f$ is almost quasi-continuous.

Then $f$ is quasi-continuous.

Proof. Similar to in the proof of Theorem 22, one can establish that $f$ is cliquish. By (2), it follows that $f$ is quasi-continuous.

Acknowledgment. The authors express their gratitude to the referees for useful comments which helped to improve the original version of this article. 


\section{References}

[1] J. Borsík and J. Doboš, On decomposition of quasicontinuity, Real Anal. Exchange., 16(1) (1990/91), 292-305.

[2] A. M. Bruckner and J. C. Ceder, Darboux continuity, Jahresber. Deutsch. Math.-Verein., 67 (1964/65), 93-117.

[3] Z. Frolik, Remarks concerning the invariance of Baire spaces under mappings, Czech. Math. J., 11(3) (1961), 381-385.

[4] L. A. Fudali, On cliquish functions on product spaces, Math. Slovaca., 33(1) (1983), 53-58.

[5] R. G. Gibson, Concerning a characterization of continuity, Real Anal. Exchange, 22(1) (1996/97), 437-442.

[6] T. Husain, Almost continuous mappings, Prace Math., 10 (1966), 1-7.

[7] S. Kempisty, Sur les fonctions quasicontinues, Fund. Math., 19 (1932), 184-197.

[8] V. I. Kretsu and V. K. Maslyuchenko, Stallings continuity, separate continuity and closed graph functions, Nauk. Visn. Chernivets'kogo Univ., Mat., 349 (2007), 50-54. (Ukrainian).

[9] N. Levine, A decomposition of continuity in topological spaces, Amer. Math. Mon., 68 (1961), 44-46.

[10] S. Marcus, Sur les fonctions quasicontinues au sens de S. Kempisty, Colloq. Math., 8 (1961), 47-53.

[11] V. K. Maslyuchenko and V. V. Nesterenko, Decomposition of continuity and transition maps, Mat. Visn. Nauk. Tov. Im. Shevchenka, 8 (2011), 132-150. (Ukrainian).

[12] M. Matejdes, Continuity points of functions on product spaces, Real Anal. Exchange, 23(1) (1997/98), 275-280.

[13] R. A. Mimna, A note on separate continuity and connectivity properties, Math. Bohem., 122(1) (1997), 57-61.

[14] R. Mimna and D. Rose, A note on closed graph functions and local $w^{*}$ continuity, Real Anal. Exchange, 18(2) (1992/93), 549-552. 
[15] R. Mimna and D. Rose, On local relative continuity, Real Anal. Exchange, 20(2) (1994/95), 823-830.

[16] V. Popa, Asupra unor forme slabite de continuitate, St. Cerc. Mat., 33 (1981), 543-546.

[17] V. Pták, On complete topological linear spaces, Czech. Math. J., 3(78) (1953), 301-364. (Russian).

[18] J. Smital and E. Stanova, On almost continuous functions, Acta. Math. Univ. Comenian, 37 (1980), 147-155.

[19] B. D. Smith, An alternate characterization of continuity, Proc. Amer. Math. Soc., 39 (1973), 318-320.

[20] J. Stallings, Fixed point theorems for connectivity maps, Fund. Math., 47 (1959), 249-263.

[21] H. P. Thielman, Types of functions, Amer. Math. Monthly, 60 (1953), $156-161$. 